\title{
LES TESTS D'EXPLORATION DE LA FECONDANCE DES SPERMATOZOIDES EN VUE D'UNE FECONDATION ASSISTEE
}

\author{
Patrick Fénichel*
}

Laboratoire de Biologie de la Reproduction et de FIV, Faculté de Médecine, 06107 Nice Cedex 2

\begin{abstract}
FERTILIZING ABILITY TESTING IN ASSISTED FERTILIZATION. It is now possible to evaluate each specific function of the spermatozoon by diagnostic testing. These tests, which are correlated with the outcome of male factor IVF, are unnecessary in routine practice. However, they are highly relevant in cases of repeated fertilization failure at IVF, allowing the detection of a specific defect in one of the component processes of fertilization which facilitates the decision as to whether to either abandon further attempts or continue with assisted fertilization treatment. Such testing must allow selection of the appropriate sperm preparation and microinjection methods and also clarify the indications, contraindications and risks of these techniques which, for the present, remain in the research domain. Key words : Assisted fertilization - gametic interaction - videomicrography - hemi-zona test - acrosome reaction - hamster test - nuclear decondensation. Andrologie, 1992, 2 : 58-60.
\end{abstract}

\section{INTRODUCTION}

Le développement des procréations médicalement assistées a bouleversé ces dix dernières années l'approche diagnostique et thérapeutique des stérilités masculines. En particulier la fécondation in vitro (FIV), en réalisant les conditions d'un test de fécondance objectif, a mis en exergue le caractère insuffisant des paramètres classiques du spermogramme, très inconstamment corrélés à la fécondance (34), et la nécessité d'aborder l'exploration de la fertilité masculine par une approche plus fonctionnelle et moins morphologique à partir des cinq grandes fonctions de cette cellule hyperspécialisée qu'est le spermatozoide. Ces cinq fonctions sont successivement: le transport, possible grâce au mouvement flagellaire, la reconnaissance ovocytaire par l'intermédiaire d'un récepteur membranaire pour la glycoprotéine ZP3, l'activation membranaire avec la capacitation survenant au cours du trajet dans les voies féminines puis la réaction acrosomique au contact de la zone pellucide, la fonction fusiogène et d'activation ovocytaire après une phase de reconnaissance / adhésion et enfin l'aptitude du noyau à se décondenser au sein du cytoplasme ovocytaire. Non seulement des tests existent aujourd'hui pour explorer ces différentes fonctions, mais la biochimie et la biologie moléculaire laissent entrevoir la possibilité de démembrer des anomalies spécifiques responsables d'hypofertilité ou de stérilité et tou- chant sélectivement l'une ou plusieurs de ces fonctions.

La FIV a permis également, d'un point de vue thérapeutique, de court-circuiter certaines de ces étapes de la fécondation comme le tri à travers la glaire cervicale, le transport et la capacitation, remplacés par l'incubation dans un milieu de culture et par la sélection d'une sous-population hypermobile. Plus récemment la fécondation assistée (FA) a introduit d'autres bouleversements: d'une part elle permet de court-circuiter de nouvelles étapes comme celle de la reconnaissance spécifique de la zone pellucide et sa pénétration, dans le cadre du zona - drilling et de l'injection sous la zone pellucide (SUZI), ou comme celle de l'adhésion / fusion en cas d'injection directe intra-cytoplasmique, et d'autre part elle conduit à la sélection non plus d'une sous-population, mais de quelques, voire d'un seul, spermatozoide. Les indications, les contreindications, les risques et les résultats de ces techniques ne sont pas encore réellement connus; il semble donc raisonnable d'appliquer avant toute tentative ces tests de fécondance,afin de tirer le maximum de renseignements des études préliminaires en cours. Le but de cette exploration sera triple :

1) comprendre l'échec des tentatives répétées de FIV en découvrant l'anomalie spécifique si elle existe, permettant d'indiquer ou de contre-indiquer la fécondation assistée.

2) Choisir la méthode de fécondation assistée la plus adéquate.

3) Permettre la sélection des spermatozoides préparés dans des conditions appropriées.

\section{LE TRANSPORT}

L'analyse de la mobilité des spermatozoides, qu'elle soit simple (\% de spermatozoides mobiles avant ou après séparation du liquide séminal, test de pénétration dans la glaire) ou plus objective, par vidéomicrographie assistée par ordinateur, permet de donner une indication pronostique avant une tentative de FIV (16). Après échecs répétés de FIV, elle permet d'apprécier objectivement une anomalie spécifique du flagelle qui sera confirmée par microscopie électronique (dyskinésies structurales axonémales ou péri-axonémales), de constater que globalement les paramètres sont très inférieurs à la normale, avec en particulier un taux très bas d'amplitude latérale de la tête ou de vitesse curvilinéaire avant et /ou après pré-incubation dans un milieu capacitant, traduisant l'inaptitude à acquérir un mouvement hyperactivé. Enfin elle confirme parfois que tous les paramètres du mouvement sont normaux. Ainsi Feneux et coll. (9), dans une série de 48 non fécondances répétées inexpliquées, retrouvent-ils dans 15 cas une anomalie de la fonction cinétique, simple, ou associée, dont 3 dyskinésies structurales.

La constatation d'une anomalie importante de la fonction cinétique pourrait théoriquement justifier le recours à une technique de fécondation assistée afin de court-circuiter au moins la pénétration à travers la zone pellucide comme le proposent Wolf et Jouannet (42). Une fécondation a pu être obtenue par l'injection sous la zone pellucide de plusieurs spermatozoides d'un sujet porteur d'un syndrome de Kartagener (27), voire même d'un seul spermatozoide immobile (3), laissant supposer qu'une bonne mobilité ne serait pas nécessaire pour permettre la fusion intergamétique et la décondensation du noyau. Des résultats semblables ont été obtenus en injectant directement le spermatozoide dans l'ovocyte de hamster (2) ou l'ovocyte humain (18). Néanmoins, en terme de résultats, il sera nécessaire de pouvoir corréler la mobilité de la population utilisée ainsi que celle des ou du spermatozoide microinjecté (s).

\section{LA LIAISON A LA ZONE PELLUCIDE}

Depuis les travaux de Saling (35), on sait que la membrane plasmique de la tête du spermatozoide possède des récepteurs spécifiques pour la glycoprotéine de la zone pellucide ZP3, et que cette liaison constitue une des étapes principales de l'interaction gamétique. Overstreet et coll. (30) ont les premiers attiré l'attention sur l'absence possible de liaison des spermatozoides à la zone pellucide dans certains cas d'infertilité masculine. L'absence totale, ou la faible liaison à la zone pellucide, peut déja être observée et notée lors des tentatives infructueuses de FIV. Deux types de test de liaison à la zone pellucide ont été proposés. Overstreet (30), puis d'autres auteurs $\{7,28)$, ont utilisés des ovocytes humains non fécondés obtenus sur ovaires de cadavre ou après échecs de FIV. Ces ovocytes peuvent être conservés très facilement sans altération de la zona dans une solution hypertonique contenant 1 à $1,5 \mathrm{M}$ de chlorure ou de sulfate de magnésium $(25,28)$. L'incubation d'une quantité égale de spermatozoides témoins et à tester permet, si ces spermatozoides ont été marqués differemment par un fluorochrome, de calculer un index de liaison à la zone pellucide (28). Burkman et coll. (4) ont développé une autre approche dans leur hémi-zona test qui consiste à couper en deux l'ovocyte à l'aide d'un micromanipulateur, et à comparer sur ces deux 
moitiés équivalentes la liaison d'un sperme témoin et à tester, conduisant également à un index de liaison. Ces tests ont une bonne valeur prédictive en FIV tout venant $(28,30)$ ou en cas de tératospermie (25). Ils permettent de dépister en cas de non fécondance répétée inexpliquée en FIV, une anomalie spécifique de la liaison à la zona (28), qui constituerait l'indication la plus logique de l'injection sous la zone pellucide.

\section{LA CAPACITATION}

Acquise au cours du trajet à travers les fluides du tractus génital féminin, nécessaire à l'acquisition du pouvoir fécondant, la capacitation n'a pourtant pas de substratum morphologique, même ultrastructural, lui permettant d'être visualisée. Elle implique en fait des modifications biochimiques membranaires et cytoplasmiques encore imparfaitement identifiées et difficiles à mettre en évidence. Pour l'apprécier, deux tests indirects ont été proposés: le hamster-test que nous reverrons plus loin, qui nécessite une pré-capacitation, et qui a donc été utilisé indirectement par Perreault et Rogers (33) pour mettre en évidence la grande variabilité inter-individuelle, même si le temps moyen de capacitation a été considéré comme étant de l'ordre de 6 heures pour l'espèce humaine. La réponse aux ionophores calciques en terme de réaction acrosomique dépend du temps de pré-incubation dans les milieux dits "capacitants". Elle a été proposée également par Byrd et Wolf (5) et par Fénichel et coll. (11) pour apprécier, toujours indirectement, l'aptitude d'un sperme à capaciter. Deux autres méthodes cherchent à visualiser plus directement les modifications membranaires liées à la capacitation, à l'aide de la chlortétracycline pour Lee et coll. (23), et d'anticorps antiphosphotyrosine pour Naz et Coll. (26). L'absence de réponse au ionophore retrouvé dans plusieurs cas de non fécondance répétée et inexpliquée par Fénichel et Coll. (12) peut être en rapport avec un retard de capacitation. Cette capacitation demeure nécessaire dans la préparation des spermatozoides en vue d'une fécondation assistée par zona-drilling ou SUZI. L'intérêt d'utiliser des activateurs de la'capacitation (milieu riche en albumine, liquide folliculaire, progestérone) reste à démontrer.

\section{LA REACTION ACROSOMIQUE (RA)}

Phénomène d'exocytose calcium-dépendant, survenant au contact de la zone pellucide, la RA met en jeu toute une cascade d'évènements membranaires et post-membranaires équivalent à une activation cellulaire. Elle permet la libération d'enzymes favorisant la pénétration de la zona comme l'acrosine, et la modification de la plaque équatoriale permettant la fusion avec la membrane ovocytaire. Troix types d'approche de l'acrosome ont été corrélées avec la fécondance des spermatozoides, et ont mis en évidence l'importance de cette étape au cours de la fécondation :

a) L'étude morphologique de l'acrosome à l'aide de sondes fluorescentes (lectines (24) ou anticorps monoclonal (14)), ou de la microscopie électronique
(10), dans la FIV toutes indications confondues (14), en cas de tératospermie supérieure à 70\% (24), et en cas de non fécondance répétée inexpliquée (10).

b) Le taux spontané de RA qui, lorsqu'il est trop élevé, semble de mauvais pronostic $(9,12,13,27)$.

c) Le taux induit de RA qui a été retrouvé avec le ionophore corrélé à la fécondance (9), et abaissé en cas d'échecs inexpliqués de FIV $(12,37)$, et avec le liquide folliculaire diminué en cas de stérilité inexpliquée (6).

La microinjection de spermatozoides non acrosome-réagis sous la zone pellucide (36), ou de spermatozoides microcéphales à tête ronde sans acrosome (19), ne permet pas d'obtenir une fécondation. Par contre de tels spermatozoides anormaux microinjectés directement dans le cytoplasme ovocytaire sont susceptibles d'entrainer une décondensation du noyau, voire une segmentation (19). Il parait donc souhaitable dans le cadre de la fécondation assistée (zona-drilling ou SUZI), de favoriser l'induction de la réaction acrosomique lors de la préparation des spermatozoides comme l'indique l'étude de Sathananthan et coll. (36). Plusieurs auteurs ont ainsi montré une amélioration des résultats après induction de la RA par différents procédés d'abord chez l'animal (17), puis avec des spermatozoides humains injectés sous la pellucide d'ovocytes de hamster (20), enfin plus récemment en fécondation humaine $(31,40)$. Plusieurs inducteurs ont été proposés dont le liquide folliculaire (31), l'électroporation(31, 40), la progestérone (32) ou le strontium (22). Il est nécessaire que leur action soit proche de la physiologie et qu'elle n'altére pas la qualité des spermatozoides. L'idéal serait de pouvoir disposer de zones pellucides (39) ou d'un recombinant de ZP3, ce qui ne devrait pas tarder.

Si les premières fécondations obtenues après SUZI l'ont été à partir de plusieurs spermatozoides micro-injectés et au prix d'un pourcentage non négligeable de polyspermie $(20,21,423)$, on sait que l'injection d'un seul spermatozoide peut être suffisante (22). Il est donc souhaitable d'enrichir la population de spermatozoides préparés en vue d'une fécondation assistée en cellules ayant accompli la RA, puis d'essayer de les sélectionner. La récupération de spermatozoides acrosome-réagis peut se concevoir à l'aide d'anticorps monoclonaux de souris qui se fixent sélectivement sur la membrane interne de l'acrosome $(13,29)$ et qui sont reconnus par des immunobilles dirigées contre les immunoglobulines de souris. L'utilisation de billes magnétiques permet de réunir ces spermatozoides (29) à l'aide d'un aimant: reste à savoir si les spermatozoides alors détachés des billes sont en bon état et toujours fécondants.

\section{LA FUSION INTERGAMETIQUE}

Une fois pénétré à travers la zone pellucide, le spermatozoide va fusionner avec la membrane ovocytaire. Cette étape comprend des phases de recon- naissance, d'adhésion puis de fusion qui mettent en jeu la plaque équatoriale et la cape post-acrosomique, mais dont les bases moléculaires sont encore imprécises.

Le test de pénétration croisée des spermatozoïdes humains dans l'ovocyte dépellucidé de hamster constitue actuellement le test de référence concernant cette étape (15). Ce test proposé par Yanagimachi et coll. (43) en 1978 a longtemps été considéré comme le test de fécondance par excellence en laboratoire. Puis des discordances apparentes et des corrélations approximatives ont ensuite jeté un certain discrédit sur ce test. En fait il est nécessaire de bien considérer les fonctions évaluées lors de ce test, à savoir: la capacitation, la réaction acrosomique, la fusion intergamétique et la décondensation des noyaux (15). C'est d'ailleurs la raison pour laquelle il a été proposé d'optimaliser le test en induisant la RA à l'aide de calcium ionophore ou du milieu d'incubation hyperosmolaire (1). Pour interpréter ce test, il convient donc d'apprécier d'abord la décondensation des noyaux ; en leur absence, la liaison des spermatozoides à la membrane ovocytaire, puis le taux de RA, voire l'état de capacitation des spermatozoïdes testés. En cas de test négatif i] peut être intéressant de vérifier à l'aide de la microscopie électronique (10), ou en immunofluorescence à l'aide d'un anticorps monoclonal dirigé contre une protéine du cytosquelette au niveau de la plaque équatoriale, comme la calicine (8), s'il existe des anomalies de la zone fusiogène. Il pourrait également être utile de vérifier l'aptitude des noyaux à décondenser lors de leur microinjection directe dans l'ooplasme.

Le résultat de ce test parait capital dans l'exploration d'un échec inexpliqué de FIV et/ou dans l'alternative d'une fécondation assistée. A quoi servirait le zona-drilling ou la SUZI si la fusion n'était pas possible?

\section{LA FONCTION NUCLEAIRE}

La condensation nucléaire est essentielle pour la fonction du spermatozoïde qui consiste à apporter le génome paternel dans le cytoplasme ovocytaire. Cette condensation peut être apprécier par la coloration nucléaire au bleu d'aniline de Terquem et Dadoune (38), qui ne colore que les noyaux immatures imparfaitement condensés. Un taux élevé de noyaux immatures a été retrouvé associé aux anomalies morphologiques des spermatozoides, ou en cas de non fécondance inexpliquée $(10,28)$. Néanmoins, avant d'envisager une fécondation assistée, l'élément utile ici est de savoir si le noyau est susceptible de se décondenser. Une microinjection directe dans l'ovocyte de hamster serait donc théoriquement indiquée.

\section{CONCLUSION}

Il est actuellement possible d'explorer de manière spécifique chaque étape de la fécondation humaine. Ces tests d'une difficulté, d'une faisabilité et d'un coût variables, ne sauraient être utilisés en routine même s'ils ont été généralement corrélés 
globalement aux résultats de FIV. Par contre en cas d'échecs répétés en FIV pour indication masculine, soit apparemment inexpliquée, soit associée à une altération du spermogramme, ces tests peuvent être entrepris (hormis en cas d'azoospermie ou d'oligospermie extrême). Ils devraient permettre 1) de dépister, si elle existe, une anomalie spécifique à l'origine de l'hypofertilité, 2) de décider ou non de la poursuite de la FIV dans des conditions particulières, ou d'indiquer le passage à une fécondation assistée ou l'abandon avec recours éventuel à un sperme de donneur, 3) de choisir en cas de fécondation assistée le mode de sélection et de microinjection des spermatozoides. Ainsi il parait illusoire en cas d'anomalie fusiogène d'injecter les spematozoides sous la pelilucide, et il est préférable d'induire la RA avant le SUZI. Les méthodes d'induction et d'évaluation de la RA sont donc très utiles dans ce cas, et le seraient encore plus si l'on pouvait sélectionner un seul spermatozoide réagi, permettant à la fois d'augmenter les chances de succès et de diminuer les risques de polyspermie.

$\mathrm{Ni}$ les indications, ni les résultats, ni les risques des méthodes de fécondation assistée ne paraissent pour l'instant bien évalués $(21,28,31,41,42)$. Une des particularités de la fécondation assistée est de diminuer encore, en court-circuitant les barrières, la sélection naturelle des spermatozoides, voire de la supprimer comme dans l'injection intra-ovocytaire, et d'opérer elle-même de façon plus ou moin arbitraire le tri. Ce tri, dont les conditions devraient pouvoir peu à peu être optimalisées, constitue à la fois son avantage et son potentiel danger. C'est la raison pour laquelle ces tests nous paraissent devoir être réalisés systématiquement avant toute tentative, afin d'en tirer le maximum de renseignements pour l'avenir $(28,42)$.

\section{REFERENCES}

1 - Aitken R.J., Thatcher S., Glasier A.F. et al: Relative ability of modified versions of the hamster oocyte penetration test incorporating hyperosmotic medium or the ionophore A23187, to predict IVF outcome. Human Reproduction, 1987, 2: 227-231.

2 - Aitken R.J, Ross A., Lees M.M. et al. : Analysis of sperm function in Kartagener's syndrome. Fertil Steril, 1983, 228 : 195201 .

3 - Bongso T.A., Sathananthan A.H., Womg P.C. et al. : Human fertilization by microinjection of immotile spermatozoa. Human Reprod, 1989, 4: 175- 179.

4 - Burkman L.J., Coddington C.C., Franken D.R. et al. ; The hemizona assay HZA: development of the diagnostic test for binding of human spermatozoa to the human hemizona pellucida to predict fertilization potential. Fertil Steril, 1988; 49: 688-697.

5 - Byrd W. Wolf D.P. : Acrosomal status in fresh and capacitated human ejaculated spenm. Biol Reprod, 1986, 34: 859-869.

6 - Calvo L., Vantman D., Banks S.M. et al. : Follicular fluid induced acrosome reaction distinguishes a subgroup of men with unexplained infertility not identified by semen analysis. Fertil Steril, 1989, 52 : 1048-1054

7 - Cordonnier I.L., Grignon $G$. : Tests de fécondance d'un sperme humain, remise à jour de l'expérience d'Overstreet: étude de 309 spermes. Contr. Fertil Sex., 1989, 17; 736-737.

8 - Courtot A.M. La membrane du spermatozoide et la fusion gamétique. Contracept Fertil. Sex ; 1990, 18, 549-551.

9 - Cummins J.M. : Evaluation of acrosomal function as a test for human sperm fertilizing competence, In Comparative spermatology 20 years after. B.Baccetti Ed., Raven Press, Volume 75, New York, 1992 pp 821-824

10 - Feneux D., Jouannet P. : Explorations en cas de non fécondance inexpliquée des spermatozoides. Contr. Fertil Sex., 1991, 19: 128-133.

11 - Fénichel P., Donzeau M., Hsi B.L. et al. : Le ionophore A23187: un tets de fécondance du sperme avant FIV? Andrologie, 1991, 1:15-16.

12 - Fénichel P. Donzeau M., Farahifar D. et al. : Dynamics of human sperm acrosome reaction: relation with in vitro fertilization. Fertil Steril, 1991, 55: 994-999.

13 - Fénichel P : Tests d'exploration de la fonction acrosomique: application en infertilité masculine. Contr. Fertil Sex, 1991, 19 : $811-818$.

14 - Gallo J.M., Escalier D., Grellier P. et al. Characterization : of a monoclonal antibody to human proacrosin and its use in acrosomal status evaluation. J. Histochem, Cytochem, 1991, 39: 273282

15 - Gould J.E., Overstreet J.W., Yanagimachi H. et al. : What functions of the sperm cell are measured by in vitro fetilization of zona-free hamster eggs? Fertil. steril 1983, 40:344-351.

16 - Guérin J.F. : Méthodes d'analyse de la mobilité des spermatozoides: intérêt dans un bilan de fertilité masculine. Contr. Fertil. Sex. , 1991, 19: 81-85.

17 - Lacham 0., Trounson A., Holden C. et al. : Fertilization and development of mouse eggs injected under the zona pellucida with single spermatozoa treated to induce the acrosome reaction. Gamete Res, 1989, 23: 233-243.

18 - Lanzendorf S.E., Maloney M.K., Veeck L.L. : A preclinical evaluation of pronuclear formation by microinjection of human spermatozoa into human oocytes. Fertil. Steril, 1988, 49: 835842

19 - Lanzendorf S.E., Maloney M.K., Ackerman S, et al.: Fertilizing potential of acrosome-defective sperm following microsurgical injection into eggs. Gamete Res, 1988, 329-337.

20 - Lassale B., Courtot A.M., Testard J. : In vitro fertilization of hamster and human oocytes by microinjection of human sperm. Gamete Res, 1987, 16: 69-75.

21 - Laufer N., Simon A. : Treatment of male infertility by gamete micromanipulation. Human Reproduction, 1992, 7:73-80.

22 - Laws-King A., Trounson A., Sathananthan $\mathrm{H}$. et al : Fertilization of human oocytes by microinjection of a single spermatozoon under the zona pellucida. Fertil Steril, 1987, $48: 637$ 642 .

23 - Lee M.A., Trucco G.S., Bechtol K.B. et al. : Capacitation and acrosome reaction in human spermatozoa monitored by a chlortetracycline fluorescence assay, Fertil. Steril, 1987, 48: 649658

24 - Liu D.Y., Gordon-Baker H.W. : The proportion of human sperm with poor morphology but normal intact acrosomes detected with pisum sativum agglutinin correlates with fertilization in vitro. Fertil Steril 1988, 50: 288-293.

25 - Liu D.Y., Clarke G.N., Lopata A. et al. : A sperm-zona pellucida binding test and in vitro fertilization. Fertil. Steril, 1989, 52: $281-287$.

26 - Naz R.K., Ahmad K., Kumar R. : Role of membrane phosphotyrosine proteins in human spermatozoal function J. Cell. Sci 1991, 99: 157-163.

27 - Ng S.C., Sathananthan A.H., Edririsinghe W.R.: Fertilization of a human egg with sperm from a patient with immotile cilia syndrome: case report . In Advances in Fertility and Sterility, SS Ratnam, ES Teoh, C Anandakumar eds, Lancaster, Parthenon Publishing, 1987, p71.

28- Oehninger S., Acosta A.A., Veeck L.L. et al. : Recurrent failure of in vitro fertilization: role of the hemizona assay in the sequential diagnosis of specific sperm-oocyte defects. Am J Obstet Gynecol, 1991, 164: 1210-1215.

29 - Okabe M, Matzno S., Nagira M. et al. : Collection of acrosome-reacted human sperm using monoclonal antibody-coated paramagnetic beads. Molecul Reprod Dev, 1992, 32: 389-393.
30 - Overstreet J.W., Yanagimachi R., Katz D.F. et al. Penetration of human spermatozoa into the human zona pellucida and the zona-free hamster egg: a study of fertile donors and infertile patients. Fertil. Steril, 1980, 33: 534-540.

31 - Palermo G., Joris H., Devroey P. et al. ; Induction of acrosome reaction in human spermatozoa used for subzonal insemination. Human Reproduction, 1992, 7: 248-254.

32 - Parinaud J., Labal B., Vieitez G. : High progesterone concentrations induce acrosome reaction with a low cytotoxic effect.Fertil. Steril, 1992 sous presse.

33 - Perreault S.D., Rogers B.J. : Capacitation pattern of human spermatozoa. Fertil. Steril, 1982, 38: 258-263.

34 - Polansky F.F., Lamb E.J. Do the results of semen analysis predict future fertility? A survival analysis study. Fertil. Steril, 1988; 49: 1059-1065.

35 - Saling P. : Mammalian sperm interaction with extracellular matrix of the egg: In : Reviews of Reproductive Biology, SR Milligan ed, Oxford. $1989: 339-370$

36 - Sathananthan A.H., Ng S.C., Trounson A., et al. : Human micro-insemination by injection of single or multiple sperm: ultrastructure. Human Reproduction, 1989, 4: 574-581.

37 - Takahashi K., Wetszels A.M.M., Goverde H.J.M. et al. : The kinetics of the acrosome reaction of human spermatozoa and its correlation with in vitro fertilization Fertil. Steril, 1992, 57: 889. 894

38 - Terquem A., Dadoune J.P. : Aniline blue staining of human spermatozoa chromatin. Evaluation of nuclear maturation. In The sperm Cell, J. André ed, Martinus Nijjhoff Publishers, The Hague, pp.249-252.

39 - Tesarik J. : Comparison of acrosome reaction - inducing activities of human cumulus oophorus, follicular fluid and ionophore A23187 in human sperm populations of proven fertilizing ability in vitro. J. Reprod. Fert, 1985, 74: 383-388.

40 - Tomkins P.T., Houghton J.A. The rapid induction of the acrosome reaction of human spermatozoa by electropermeabilization. Fertil steril 1988, 50: 329-336.

41 - Vanderzwalmen P., Barlow P., Nijs M. et al. : Usefulness of partial dissection of the zona pellucida in a human in-vitro fertilization programme. Human Reproduction, 1992, 7: 337-344.

42 - Wolf J.P., Jouannet P. : Intérêt des méthodes de microfécondation dans l'infertilité masculine. Contr. Fertil, Sex., 1991, 19: $168-176$.

43 - Yanagimachi R., Yanagimachi H., Rogers B.J. et al. : The use of zona - free animal ova as a test-system for the assessment of the fertilizing capacity of human spermatozoa Biol. Reprod, 1976, 15: 471-479.

RESUME : Il est possible actuellement d'évaluer chaque fonction spécifique du spermatozoîde à l'aide de tests d'exploration. Ces tests, bien que corrélés aux résultats de la FIV d'indication masculine, ne sont pas nécessaires en pratique courante. Par contre ils prennent toute leur signification en cas d'échecs répétés en FIV afin de rechercher une anomalie spécifique d'une des étapes de la fécondation et pour décider l'arrêt définitif des tentatives ou le recours éventuel à une fécondation assistée. Ils devraient permettre de choisir la technique de préparation et de microinjection du ou des spermatozoides et de clarifier les indications, les contreindications et risques de ces techniques qui restent pour l'instant du domaine de l'expérimentation. Mots clés : Fécondation assistée - interaction gamétique - videomicrographie assistée par ordinateur - hémizona test - réaction acrosomique - hamster test - décondensation nucléaire. Andrologie, 1992, 2 : 58-60. 